\title{
COMPETENCY FOR GRAVIRESPONSE IN THE LEAF-SHEATH PULVINUS OF AVENA SATIVA: ONSET TO LOSS'
}

\author{
Thomas G. Brock ${ }^{2}$ And Peter B. Kaufman \\ Department of Biology, University of Michigan, Ann Arbor, Michigan 48109
}

\begin{abstract}
A BST RACT
The development of the leaf-sheath pulvinus of oat (Avena sativa L. cv. Victory) was studied in terms of its competency to respond to gravistimulation. Stages of onset of competency, maximum competency and loss of competency were identified, using the length of the supertending internode as a developmental marker. During the early phases in the onset of competency, the latency period between stimulus and graviresponse decreased and the steady state response rate increased significantly. When fully competent, the latency period remained constant as the plant continued to develop, suggesting that the latency period is relatively insensitive to quantitative changes (e.g., in carbohydrate or nutrient availability) at the cell level within the plant. In contrast, the response rate was found to increase with plant development, indicating that graviresponse rate is more strongly influenced by quantitative cellular changes. The total possible graviresponse of a single oat pulvinus was confirmed to be significantly less than the original presentation angle. This was shown to not result from a loss of competency, since the graviresponse could be reinitiated by increasing the presentation angle. As a result of the low overall graviresponse of individual pulvini, two or more pulvini are required to bring the plant apex to the vertical. This was determined to occur through the sequential, rather than simultaneous, action of successive pulvini, since a given pulvinus lost competency to gravirespond shortly after the next pulvinus became fully competent.
\end{abstract}

IN FESTUCOID GRASSES (e.g., oat, barley, wheat, rice), differential growth of the leaf-sheath pulvinus represents the main mechanism for the reorientation of the above-ground portion of the plant to a vertical position following lodging or gravistimulation (for review, see Kaufman et al., 1987). As a gravitropic organ, the leaf-sheath pulvinus is unique in a variety of ways. Unlike roots, coleoptiles, or dicot stems, it appears to function primarily as a graviresponsive organ and is not otherwise a significant contributor to elongation growth (Bridges and Wilkins, 1973; Dayanandan, Hebard, and Kaufman, 1976; Wright and Osborne, 1977). Also, both the sensing of gravity and the response to gravistimulation occur at the same site. As a result, isolated pulvini or pulvinus fragments are capable of sensing and showing a growth response to a reorientation with respect to the gravity vector (Bridges and Wilkins, 1974; Osborne and Wright, 1977). Also,

\footnotetext{
' Received for publication 19 November 1987; revision accepted 18 February 1988.

We thank James Burg and Virgie Bright for technical assistance in preparing tissue for experimentation. Special thanks also to Carol M. Fast for her enduring assistance and support throughout the study. This work was financially supported by a National Aeronautics and Space Administration Research Associate Award to TGB and through NASA grant NAGW-34 to PBK.

2 Address correspondence to this author.
}

unlike all other gravitropic systems, the reorientation of the intact plant to the vertical typically is achieved through the responses of more than one responding region: at least two pulvini, and frequently more, are involved in the graviresponse of the intact plant (Sachs, 1882; Kaufman et al., 1987).

The specialization of function of the leaf sheath pulvinus suggests that this organ would be an excellent system to study the development of gravitropic capacity. Furthermore, the ability to both perceive and respond to gravistimulation indicates that the pulvinus would be ideal for studying developmental features of these distinct phases of gravitropism. Along these lines, Wright (1986) has used pulvini of wild oat (Avena fatua) as a system to study the development of graviperception, correlating the onset of gravitropic capacity with the development of statocytes, the putative sensors of gravistimulation (Hillman and Wilkins, 1982; Song et al., 1988). Finally, the requirement of at least two pulvini to mediate the complete graviresponse of the intact plant presents the question of how the development of one pulvinus is related temporally to the responsiveness of the other.

This report presents findings from studies on the development of the pulvinus and its competency to respond to gravistimulation. In particular, the onset and development of re- 
sponsiveness are characterized in terms of the latency of response following the stimulus, the magnitude of the steady-state bending response produced by gravistimulation, and the overall graviresponse observed after $14 \mathrm{hr}$ of gravistimulation. Furthermore, the comparative development of two successive pulvini are described and indicate the kinetics of loss of capacity to respond to gravistimulation.

MATERIALS AND METHODS-Plant material-Oat plants (Avena sativa L. cv. Victory, from Svenska Allmanna A.B., Svalöf, Sweden) were raised from seed in a greenhouse with supplemental lighting to maintain a light period : dark period ratio of 18:6 hr (Kaufman, Cassell, and Adams, 1965). After approximately 4 weeks, plants were transferred to a growth chamber with the same light period: dark period ratio, $25 \pm 2 \mathrm{C}$, until plants were approximately 45 days old.

Long-term response experiments-The graviresponse of pulvini over $14 \mathrm{hr}$ was studied using isolated stem segments mounted horizontally in humid boxes at $25 \mathrm{C}$. Plants were selected for uniformity of stem diameter and straightness through the p-1 (i.e., next to last) internode. The length of the p-1 internode (IL; internode above the p-1 pulvinus) for each individual plant was noted and marked on the leaf sheath above the p-1 pulvinus. For studies involving individual pulvini, segments were excised $4 \mathrm{~cm}$ above and $4 \mathrm{~cm}$ below the p- 1 pulvinus. These dimensions were chosen since previous work indicated they were ideal for maximum gravitropic response (Kaufman, Song, and Pharis, 1985). Isolated segments were mounted in a horizontal orientation with bases in contact with wet paper towel and secured between glass plates (Dayanandan, Hebard, and Kaufman, 1976).

For studies involving two pulvini, the p-2 pulvinus (i.e., the pulvinus below the p-1 pulvinus, subtending the $\mathrm{p}-2$ internode) was used with the accompanying p-1 pulvinus. Segments were excised $4 \mathrm{~cm}$ beyond each pulvinus and taped between the pulvini to a glass plate, leaving the pulvini extending beyond the plate. Wet paper towel was placed on top of the p-2 internode (i.e., the stem segment between the p-1 and p-2 pulvini) and the assembly was placed in a humid box in the dark. Parallel experiments were performed with identically prepared tissue, except an incision was made completely through the p-2 internode, midway between pulvini, with a razor. Initial angles at all pulvini were noted and the overall graviresponse, defined as the gross segment bending response through the pulvinus, was measured as change in segment angle over $14 \mathrm{hr}$ using a protractor.

Termination of response-The capacity to reinitiate the graviresponse following an increase in presentation angle was followed using segments mounted individually with bases in vials containing $0.1 \mathrm{M}$ sucrose. Segments were given a presentation angle of $90^{\circ}$ for 5 days, followed by an increase to $115^{\circ}$ for another 3 days. Sucrose solutions were changed each day, after the angle of curvature at the pulvinus had been measured with a protractor.

Kinetics of response experiments-Graviresponse kinetics were determined using an angular recording position transducer (Metripak Corp., Cleveland, $\mathrm{OH}$ ) in series with a strip chart recorder (Kaufman and Dayanandan, 1984). Individual plants were selected for straightness of stem through the p-1 pulvinus. Length of p- 1 internode (IL) was noted and stem segments were excised $4 \mathrm{~cm}$ above and $10 \mathrm{~cm}$ below the $\mathrm{p}-1$ pulvinus. Individual segments were securely mounted in glass vials with segment bases contacting water-saturated tissue paper. The segment pulvinus and supertending stem portion were outside of the vial and free to move gravitropically. The mounted segment was initially left in a vertical orientation in the dark, $25 \mathrm{C}$, for $0.5-1.0 \mathrm{hr}$. The assembly was then rotated to a horizontal orientation and the transducer arm was placed on the leaf sheath, $1.0 \mathrm{~cm}$ distal to the $\mathrm{p}-1$ pulvinus. Latency of response was defined as the period between horizontal placement and initiation of a negative gravitropic response. Steady state rate of upward bending was defined as the continuous bending rate assumed within $6 \mathrm{hr}$ after initial negative gravitropic response and was calculated as a running average from recorder printouts, using $10 \mathrm{~min}$ increment rates over at least $3 \mathrm{hr}$.

Pulvinus measurements-Pulvinus (p-1 locus) dimensions were collected at three different times and correlated with p-1 internode length. Lengths and widths of individual pulvini were determined using a dissecting microscope fitted with a measuring ocular with a potential resolution of $0.05 \mathrm{~mm}$. Pulvinus volume, $\mathrm{V}$, was calculated as

$$
\mathrm{V}=\pi(\mathrm{L})\left(\mathrm{r}^{2}\right) \text {, }
$$

where $L$ is pulvinus length and $r$ is 0.5 times pulvinus width. Data from all three collections were pooled to provide mean dimension values for each internode increment. 


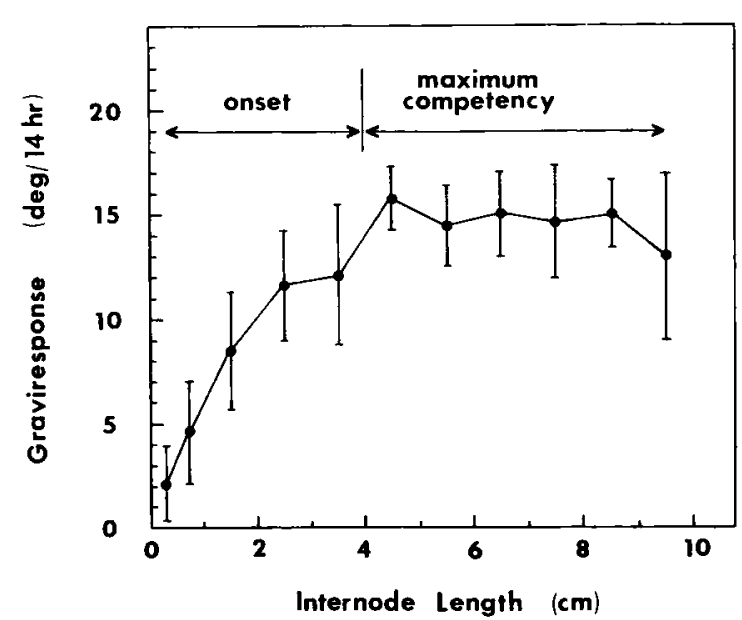

Fig. 1. Early stages of the oat pulvinus' competency to respond to gravistimulation. The change in segment angle, over $14 \mathrm{hr}$, resulting from the graviresponse of the isolated (p-1) pulvinus is correlated with plant development in terms of length of supertending (p-1) internode. Vertical lines indicate SE.

Statistical analysis-Statistical significance was determined using the nonparametric Mann-Whitney $U$ test, with $\alpha=0.01$.

RESULTS-Long-term overall graviresponse-The development of leaf-sheath pulvini can be divided into (at least) three stages with respect to competency to show a graviresponse: onset of competency and maximum competency (Fig. 1) and loss of competency (Fig. 2). Maximum overall graviresponse, in terms of change in angle over $14 \mathrm{hr}$, occurred for IL values of $4 \mathrm{~cm}$ or greater for plants grown under these conditions. For IL values less than $3 \mathrm{~cm}$, pulvini showed a reduced graviresponse, even though very young pulvini (IL less than $0.5 \mathrm{~cm}$ ) typically displayed some bending response. For IL values greater than $8 \mathrm{~cm}$, the variability in response increased with increasing IL, making the response of individual pulvini less predictable. Similar patterns were observed for $\mathrm{p}-2$ pulvini (data not presented).

There was a relatively short period when both the $p-1$ and the $p-2$ pulvini were maximally competent, and the graviresponses of both pulvini were approximately equal over $14 \mathrm{hr}$ of continuous gravistimulation (Fig. 2). This period, for IL values between 4 and $7 \mathrm{~cm}$, corresponds to 1.0 and 1.5 days in length (Kaufman et al., 1965). Before this period the p-1 pulvinus was found to be graviresponsively immature and after it the p-2 lost competency to respond. This pattern was observed whether the successive pulvini were physically isolated

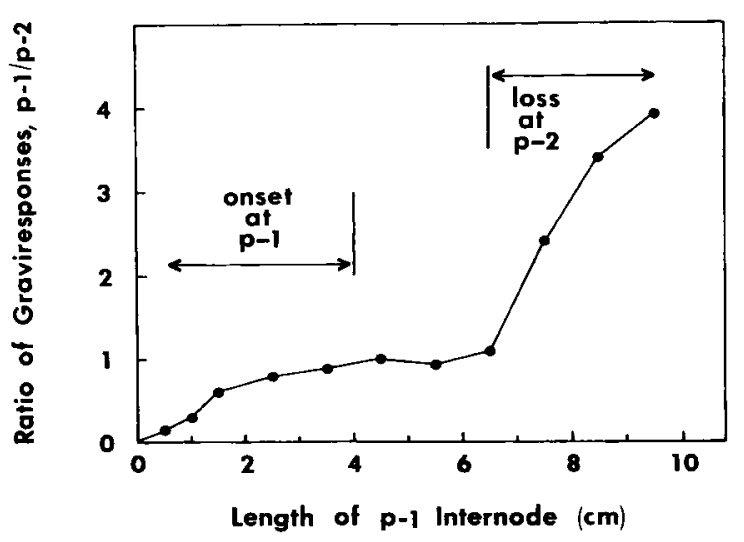

Fig. 2. Developmental stages of competency to gravirespond at two successive pulvini. The developmental relationship is presented as the ratio of the $14 \mathrm{hr}$ graviresponse of the younger ( $p-1)$ pulvinus over that of the older (p-2) one. The timing of onset of competency in the former and loss of competency in the latter is indicated.

in separate segments or they were both in one intact segment.

Termination of response-As noted above, one pulvinus alone is insufficient to bring the plant apex to a vertical orientation. A single oat pulvinus, given a presentation angle of $90^{\circ}$, can respond to generate an angle of $40-60^{\circ}$ over several days, but the response never approaches $90^{\circ}$. To test if the failure of the graviresponse to equal the presentation angle is the result of loss of competency, segments that had been gravistimulated at $90^{\circ}$ for 5 days were further gravistimulated another $25^{\circ}$ (total presentation angle $=115^{\circ}$ ). These segments displayed a rapid reinitiation of the graviresponse following the increase in presentation angle(Fig. 3 ). The change in angle induced by the additional gravistimulation was, as before, a fraction of the increase in presentation angle.

Kinetics of response-Features of the response kinetics of individual pulvini were examined to characterize pulvinus development. As young pulvini developed, the length of the latency period to initial response decreased (Fig. 4) and the steady state response rate achieved following response initiation increased (Fig. 5). During the stage of maximal competency, the latency period did not significantly change (Fig. 4). However, the steady state response rate achieved shortly after initiation of response continued to increase with increasing IL value (Fig. 5).

Change in pulvinus dimensions-Pulvinus dimensions were followed for two reasons: 1) 


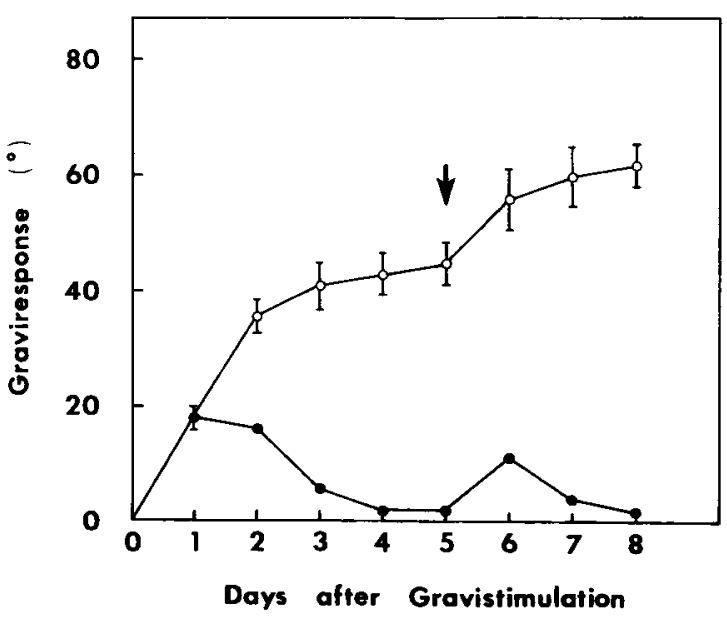

Fig. 3. The reinitiation of graviresponse following an increase in presentation angle. Initial presentation angle of $90^{\circ}$ was increased after 5 days to $115^{\circ}$ at arrow. Overall change in angle since beginning of experiment is marked by open circles $(O)$; calculated change in angle over previous $24 \mathrm{hr}$ is marked by closed circles (O). Vertical lines indicate $\mathrm{SE}$.

to determine if the maximally competent leafsheath pulvinus is nongrowing, as has been described (Arslan and Bennet-Clark, 1960), even though obvious differences in size between gravitropically incompetent pulvini and mature organs had been observed during this study, and 2) to determine if the loss of competency is associated with an increase in pulvinus diameter, which might be indicative of a role for ethylene in this stage. Oat leaf-sheath pulvini increased in length, width and volume as they became gravitropically competent (Fig.

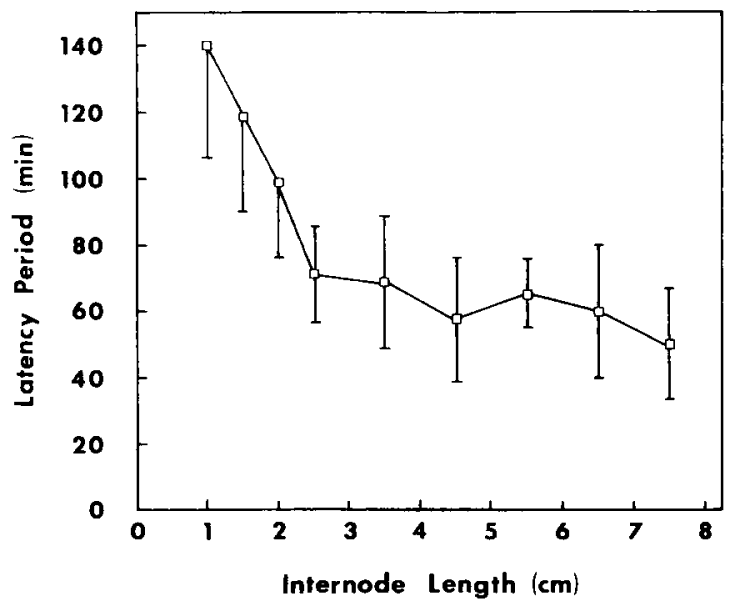

Fig. 4. Changes in the kinetics of response associated with plant and pulvinar development: mean latency period between presentation of the gravistimulus and initial negative gravitropic response. Vertical lines indicate SE.

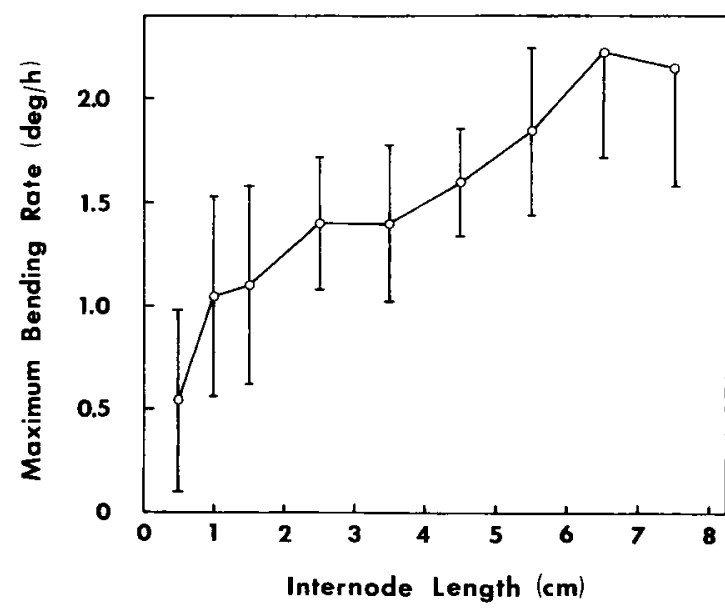

Fig. 5. Changes in the kinetics of response associated with plant and pulvinar development: mean steady state bending rate achieved within $6 \mathrm{hr}$ of graviresponse initiation. Vertical lines indicate SE.

6-8). There appeared to be a phase in pulvinus development when organ enlargement ceased temporarily. This phase was associated with the stage of maximum competency to show a graviresponse. Subsequent to this phase, pulvini resumed enlargement growth, with gains in length being comparable to those in width.

DisCusSION-When the length of the internode above a given pulvinus is used as a morphological marker for the developmental state of the plant and the subtending pulvinus, characteristics of the development of pulvinus graviresponsiveness can be analyzed and quantitatively delineated. The early stages of competency development appear to be associated with organ maturation, involving such elements as statocyte formation (Wright, 1986), as well as the parallel development of the graviresponse system. The loss of competency also appears to be a simple function of advanced development of the individual pulvini, rather than resulting from interpulvinus relationships. Possible mechanisms leading to loss of competency include increased deposition of silica (Srinivasan, Dayanandan, and Kaufman, 1979), lignification (Arslan and BennetClark, 1960), or loss of responsiveness to the stimulus (e.g., Van Volkenburgh, Schmidt, and Cleland, 1985; Brock and Kaufman, 1988).

The response of the intact plant to lodging or gravistimulation involves several pulvini. This response must be typically the result of the sequential, rather than simultaneous, responses of successive pulvini, since the time of overlap of competency of successive pulvini is short. As a result, the righting of horizontal 


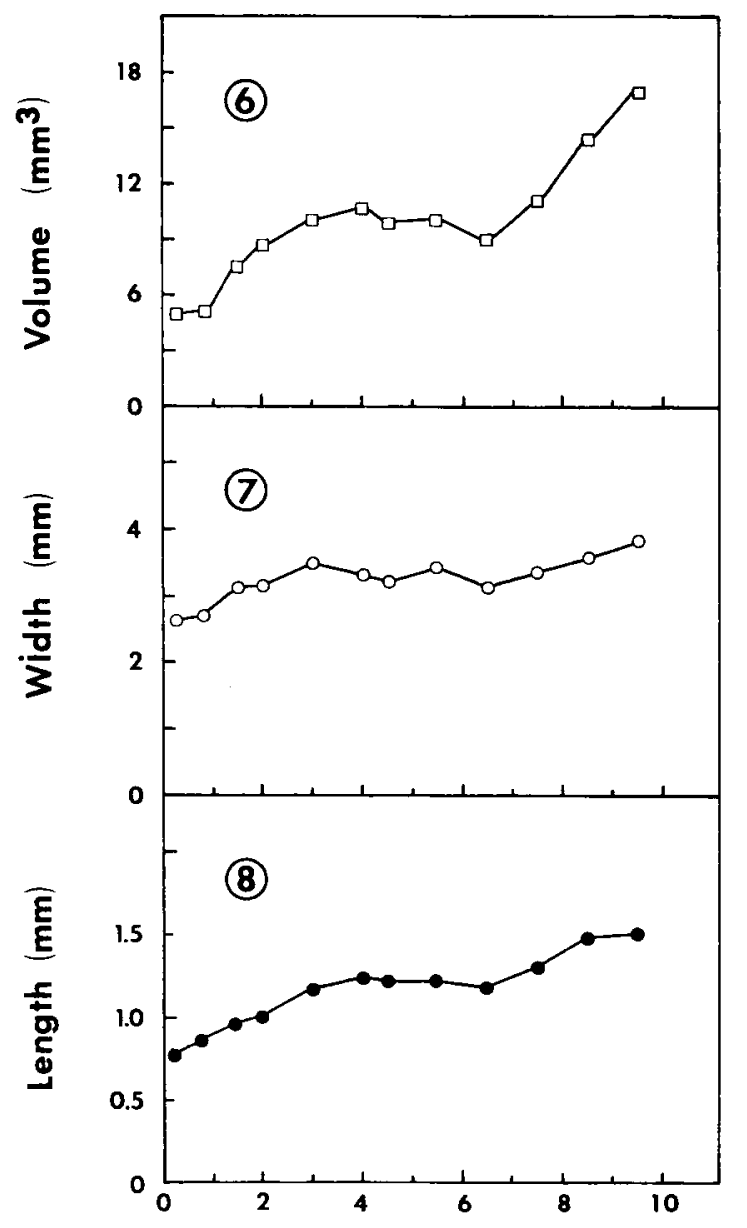

Internode Length (cm)

Fig. 6-8. Mean pulvinus dimensions correlated with plant and pulvinus development. Values were calculated from pooled data from 3 collections with 10-30 samples per point per collection. 6. Mean pulvinus volume. 7. Mean pulvinus width. 8. Mean pulvinus length.

plants may take from several days to a few weeks.

The involvement of two or more pulvini appears to be a result of design rather than necessity. That is, the growth of an individual oat pulvinus ceases before the resulting angle equals the presentation angle, and this is not due to a loss of response competency. The pulvinus is clearly competent to reinitiate the response following an increase in presentation angle (Fig. 3). Similarly, pulvini can re-respond in the opposite direction when rotated $180^{\circ}$ (Osborne and Wright, 1977), and this can be repeated several times for a single pulvinus (Kaufman et al., 1987). Also, the final response is always a fraction of the presentation angle, for a range of angles (Fig. 3; see also Brock and Kaufman, 1988).

The lack of significant change in latency period once pulvini are maximally competent suggests that initiation of response is a function of the qualitative developmental state of the organ. Once the pulvinus is gravitropically mature and all relevant components are in place, response initiation may then directly result from stimulus presentation. For example, a simplified model for pulvinus gravitropism suggests that gravistimulation causes amyloplast sedimentation, which brings membrane-bound esterases or peptidases in contact with conjugated (myo-inositol-linked or amide-linked) IAA, leading to release of IAA and subsequently to IAA-induced growth initiation (Kaufman et al., 1987). The initiation of response, in this scenario and supported by these data, should not be significantly influenced by diurnal changes, e.g., in carbohydrate or nutrient availability, at the cell or tissue level.

In contrast to this, the increase in steady state response rate associated with increasing IL indicates that response rate is strongly influenced by cellular changes, e.g., increases in substrate levels. Following the model presented above, gravity-induced response rate should be roughly analogous to hormonally-induced growth responses, which are known to be strongly influenced by carbohydrate levels and nutrient availability in oats (Adams, Kaufman, and Ikuma, 1973; Kaufman, 1983) as well as other plants (Leopold et al., 1974). This also suggests a basis for the observed enhancement of the pulvinus graviresponse by the addition of exogenous sucrose (Bridges and Wilkins, 1974; Brock and Kaufman, unpublished results).

The stationary phase in pulvinus enlargement associated with maximum competency for graviresponse points to one of the unique features of this gravitropic system: graviresponse in the pulvinus involves growth initiation and regulation of rate and duration of response. This provides a simpler model system for the study of gravitropism than organs in which the response can involve growth suppression as well as enhancement (e.g., maize root; Evans, Moore, and Hasenstein, 1986), or relocation of the growing zone (e.g., sunflower hypocotyl; Hart and MacDonald, 1984).

\section{LITERATURE CITED}

Adams, P. A., P. B. Kaufman, and H. IkUma. 1973. Effects of gibberellic acid and sucrose on the growth of oat (Avena) stem segments. Pl. Physiol. 51: 11021108.

Arslan, N., And T. A. Bennet-Clark. 1960. Geotropic behaviour of grass nodes. J. Exp. Bot. 11: 1-12. 
BRIDGES, I. G., AND M. B. WILkINS. 1973. Growth initiation in the geotropic response of wheat node. Planta 112: 191-200.

, AND M. B. WILKINS. 1974. The role of reducing sugars in the geotropic response of the wheat node. Planta 117: 243-250.

BRock, T. G., AND P. B. KaufMan. 1988. Altered growth response to exogenous auxin and gibberellic acid by gravistimulation in pulvini of Avena sativa. Pl. Physiol. 87: 130-133.

Dayanandan, P., H. V. Hebard, and P. B. Kaufman. 1976. Cell elongation in the grass pulvinus in response to geotropic stimulation and auxin application. Planta 131: 245-252.

, H. V. HeBard, V. D. Baldwin, AND P. B. Kaufman. 1977. Structure of gravity-sensitive sheath and internodal pulvini in grass shoots. Amer. J. Bot. 64: 11891199.

, AND P. B. KaUfMan. 1984. Analysis and significance of gravity-induced asymmetric growth in the grass leaf-sheaf pulvinus. Ann. Bot. 53: 29-44.

Evans, M. L., R. MOORE, AND K.-H. Hasenstein. 1986. How roots respond to gravity. Sci. Amer. 255: 112119.

HART, J. W., AND L. R. MACDonald. 1984. Is there a role for the apex in shoot geotropism? Pl. Physiol. 74: 272-277.

Hillman, S. K., and M. B. Wilkins. 1982. Gravity perception in decapped roots of Zea mays. Planta 155: 267-271.

KaufMan, P. B. 1983. Physiology and biochemistry of gibberellins in developing shoots of grasses. In S. S. Purohit [ed.], Aspects of physiology and biochemistry of plant hormones, 125-137. Kalyani Publishers, India.

, S. J. Cassell, ANd P. A. Adams. 1965. On nature of intercalary growth and cellular differentiation in internodes of Avena sativa. Bot. Gaz. 126: 1-13.

, AND P. Dayanandan. 1984. Hormonal regulation of the gravitropic response in pulvini of grass shoots. In S. S. Purohit [ed.], Physiology of plant growth and development, Vol. I, 369-385. Agro Botanical Publishers, Bikaner, India.

, I. Song, AND R. P. Pharis. 1985. Gravity perception and response mechanism in graviresponding cereal grass shoots. In S. S. Purohit [ed.], Hormonal regulation of plant growth and development, Vol. II, 189-200. Agro Botanical Publishers, Bikaner, India. , T. G. Brock, I. Song, Y. B. Rho, AND N. S. GHOSHEH. 1987. How cereal grass shoots perceive and respond to gravity. Amer. J. Bot. 74: 1446-1457.

Leopold, A. C., B. W. Poovaiah, R. K. dela Fuente, AND R. J. WILliams. 1974. Regulation of growth with inorganic solutes. In Plant growth substances 1973, 780-788. Hirokawa, Tokyo.

OSBORNE, D. J., AND M. WRIGHT. 1977. Gravity-induced cell elongation. Proc. Roy. Soc. London, Ser. B, Biol. Sci. 199: 551-564.

SACHS, J. 1882. Über Ausschliessung der geotropischen und heliotropischen Krumming wahrend des Wachsens. Arbeiten Bot. Inst. Würzburg 2: 209-225.

Song, I., C. R. Lu, T. G. Brock, AND P. B. KaufMan. 1988. Do starch statoliths act as the gravisensors in cereal grass pulvini? Pl. Physiol. 86: 1155-1162.

Srinivasan, J., P. Dayanandan, and P. B. Kaufman. 1979. Silica distribution in Equisetum hyemale var. Affine L. (Engelm.) in relation to the negative geotropic response. New Phytol. 83: 623-626.

VAN Volkenburgh, E., M. G. SChmidt, and R. E. CleLAND. 1985. Loss of capacity for acid-induced wall loosening as the principal cause of the cessation of cell enlargement in light-grown bean leaves. Planta 163: 500-505.

WrIGHT, M. 1986. The acquisition of gravisensitivity during the development of nodes of Avena fatua. $\mathbf{J}$. Pl. Growth Regul. 5: 37-47.

$\longrightarrow$, AND D. J. OSBORNE. 1977. Gravity-regulation of cell elongation in nodes. Biochem. Physiol. Pflanzen 171: 479-492. 